\title{
Perfuração facial incomum por projétil de borracha: Relato de caso
}

\author{
Unusual facial perforation by less lethal munition: Case report \\ Perforación facial inusual com bala de goma: Reporte de caso
}

\section{Resumo}

Introdução: As balas de borracha são consideradas "armas não-letais", porém, quando atingem região facial podem deixar sequelas graves ou até causar morte. Tais projéteis podem alcançar a região facial, causando destruições ósseas, danos neurológicos e vasculares, bem como, favorecer o desenvolvimento de infecções. O presente estudo tem objetivo de relatar um caso de um paciente do sexo masculino, 32 anos, acometido por um projétil compatível com bala de borracha no momento de uma dispersão policial. Relato de caso: O paciente compareceu ao serviço de emergência apresentando ptose palpebral, equimose e edema periorbitário de ambos os olhos, sendo evidenciado no olho esquerdo e ferimento corto-contuso no canto medial da órbita. Na tomografia computadorizada pré-operatória, observou-se: corpo estranho alojado na base do crânio - dentro do seio esfenoidal. O paciente foi submetido a centro cirúrgico sob anestesia geral para remoção do projétil e uso de I-PRF para fechamento de provável fistula liquórica. Discussão: As lesões penetrantes ocasionadas por bala de borracha na face devem ser tratadas por uma equipe multidisciplinar em associação com exames complementares como a tomografia computadorizada para diagnosticar e definir o melhor tratamento. Conclusão: O paciente evolui com 03 (três) meses de acompanhamento pós-operatório, sem sinais de infecção ou evidencia de fístula liquórica.

Palavras-chave: Órbita; Traumatismo craniano penetrante; Bala de borracha.

\begin{abstract}
Rubber bullets are considered "non-lethal weapons", however, when they reach the facial region they can leave serious sequelae or even cause death. Such projectiles can reach the facial region, causing bone destruction, neurological and vascular damage, as well as, favoring the development of diseases. The present study aims to report a case of a 32-year-old male patient affected by a rubber bullet-compatible projectile at the time of a police dispersion. The patient attended the emergency service of the Instituto Doutor José Frota - CE, reinforced eyelid ptosis, ecchymosis and periorbital edema of both eyes, being evidenced in the left eye and a cut-contusion wound in the
\end{abstract}


medial corner of the orbit. In the preoperative computed tomography, it was observed: foreign body housed in the blood base - inside the sphenoid sinus. The diagnosis and treatment decision was made by a multidisciplinary team. The patient was submitted to a surgical center under general anesthesia to remove the projectile and use LPR-F. The patient progresses with 03 (three) months of postoperative follow-up, with no signs of infection or outflow of CSF fistula. A search was performed in the literature for articles published in the years between 1975-2017 in English. The penetrating losses caused by a rubber bullet in the face must be treated by a multidisciplinary team in association with complementary exams such as a computed tomography to diagnose and define the treatment with a better prognosis.

Keywords: Orbit; Head injuries; Rubber bullet.

\section{Resumen}

Introducción: Las balas de goma se consideran “armas no letales”, sin embargo, cuando llegan a la región facial, pueden dejar graves secuelas o incluso causar la muerte. Dichos proyectiles pueden llegar a la región facial, provocando destrucción ósea, daño neurológico y vascular, además de favorecer el desarrollo de infecciones. El presente estudio tiene como objetivo reportar el caso de un paciente masculino de 32 años, afectado por un proyectil compatible con una bala de goma en el momento de una dispersión policial. Caso clínico: El paciente acude al servicio de urgencias con ptosis palpebral, equimosis y edema periorbitario en ambos ojos, siendo evidente en el ojo izquierdo y una herida por corte-contusión en el ángulo medial de la órbita. En la tomografía computarizada preoperatoria, se observó: cuerpo extraño alojado en la base del cráneo - dentro del seno esfenoidal. El paciente fue sometido a un centro quirúrgico bajo anestesia general para retirar el proyectil y utilizar I-PRF para cerrar una probable fístula de LCR. Discusión: Las lesiones penetrantes provocadas por una bala de goma en la cara deben ser tratadas por un equipo multidisciplinario en asociación con exámenes complementarios como la tomografía computarizada para diagnosticar y definir el mejor tratamiento. Conclusión: la paciente evoluciona con 03 (tres) meses de seguimiento postoperatorio, sin signos de infección ni evidencia de fístula de LCR.

Palabras clave: Orbita; Trauma de la cabeza; Bala de goma.

\section{Introdução}

As balas "não-letais" foram inicialmente introduzidas em Hong Kong, onde seu material consistia em madeira e eram nominadas como "disparar rodadas de bastão". O material foi substituído por borracha pelo Exército Britânico, o qual incluiu as balas de borracha em seu armamento em julho de 1970.Comumente, são utilizadas para dispersar multidões, causando ferimento não-penetrante, deixando sua impressão na pele, sendo chamado de "marcador". .(Kobayashi; Mellen, 2009)(Gross et al., 2005)(Millar et al., 1975). A face é a parte mais vulnerável do corpo a penetração após uma lesão por projétil de borracha que pode levar à morte. O terço médio da face possui estruturas ósseas finas e características anatômicas como: cavidade orbitária, que facilita a invasão da bala de borracha e ocasionar ruptura do globo ocular associada a penetração do corpo estranho no cérebro, sendo capaz de causar sequelas graves ou óbito. (Amaral et al., 2017). Lesões por bala de borracha nos seios paranasais são raras e, quando acometidos, o seio maxilar é o mais prevalente. (Gross et al., 2005)

\section{Metodologia}

O trabalho em questão aborda o caso de um paciente, de forma descritiva e qualitativa (Pereira,et al., 2018), portador de trauma maxilofacial, com o objetivo de detalhar o procedimento cirúrgico de remoção do projétil, sendo autorizado pelo paciente por meio de um Termo de Consentimento Livre e Esclarecido (TCLE) elaborado em linguagem fácil para obtenção do conhecimento acerca do destino das informações pessoais colhidas além dos riscos e benefícios para tal. O presente estudo tem por objetivo oferecer uma breve revisão da literatura, por meio da busca de artigos científicos e estudos de caso nas bases de dados MEDLINE, PubMed e Google Scholar, sobre os métodos de diagnóstico e planejamento de tratamento clínico para trauma por projétil não-letal.

\section{Relato de Caso}

Paciente do sexo masculino, 33 anos de idade, foi levado ao serviço de emergência do Instituto Doutor José Frota CE (IJF), apresentando corpo estranho em face após ser atingido por uma bala de borracha após ação policial. Paciente se 
apresentou hemodinamicante estável, Escore da Escala de Coma de Glasgow 14, sem queixas respiratórias. Ao exame clínico inicial apresentou: equimose e edema periorbitário em olho esquerdo, ferimento corto-contuso no canto interno da órbita esquerdas em sangramento ativo, ptose palpebral, epífora, amaurose em pós-trauma imediato e midríase paralítica do olho esquerdo e discreta obstrução nasal do lado esquerdo (Figura 1). Negou queixas faríngeas, otológicas e ausência de sinais clínicos compatível com paralisia facial.

Figura 1. Aspecto inicial do paciente ao ser admitido no serviço de emergência, apresentando: equimose e edema periorbitário em ambos os olhos, sendo com maior evidência no olho esquerdo (OE), ferimento corto-contuso na região medial do olho esquerdo. Em seguida, aspecto clínico após 03 meses após o procedimento cirúrgico, apresentando: cicatriz em região do canto médio, amaurose e ptose palpebral no OE.

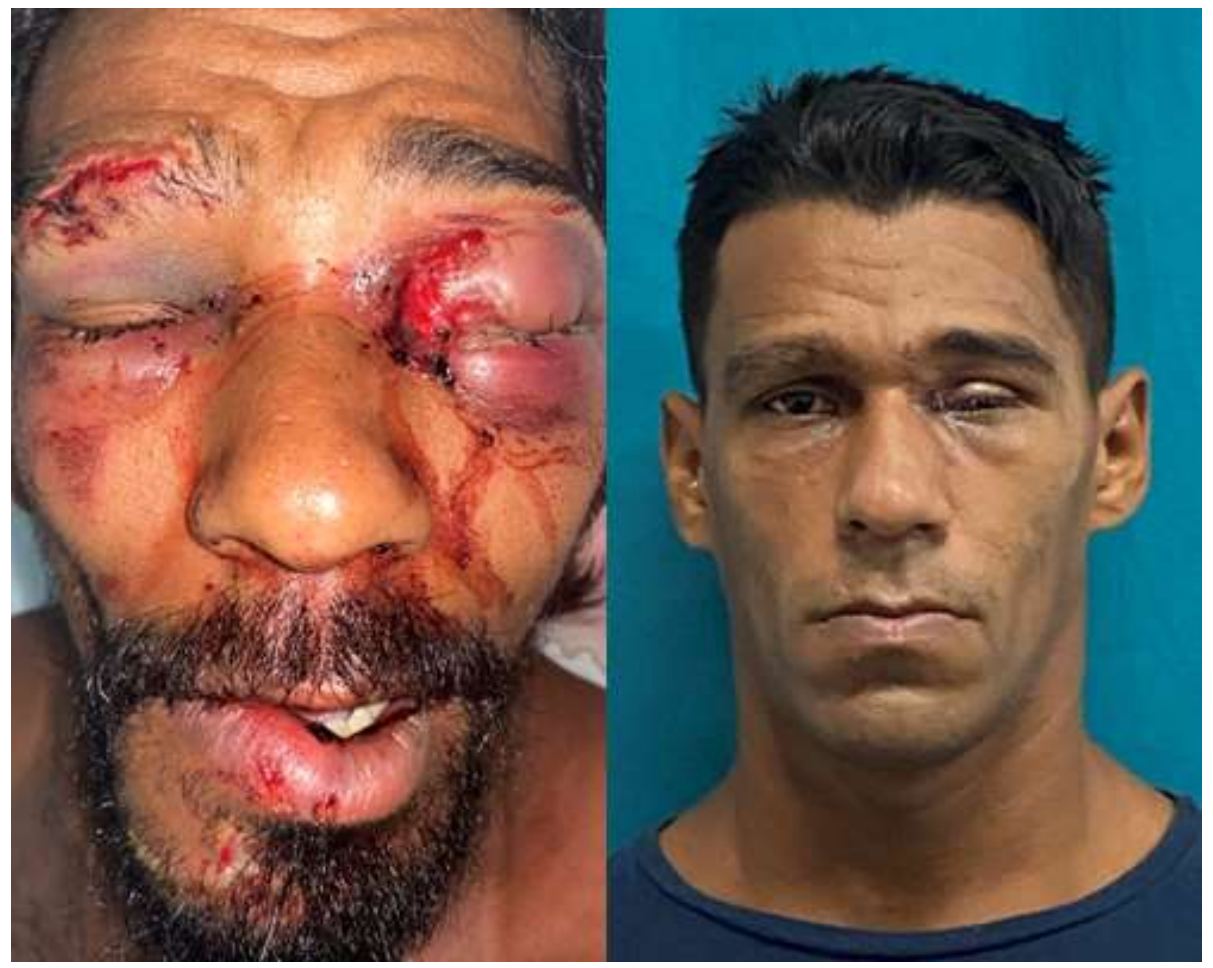

Fonte: Autores.

Na tomografia computadorizada (TC) (Figura 2), observa-se corpo estranho na região de borda medial da orbita, com fratura da parede medial da órbita, ossos próprios do nariz, seios etmoidais, ficando alojada no seio esfenoidal, com ductos lacrimais preservados e globos oculares íntegros; apresentou presença de discreto pneumoencéfalo acima da placa crivosa, sem necessidade de abordagem cirúrgica. A angiografia evidenciou não opacificação da artéria oftálmica esquerda. O paciente foi abordado em centro cirúrgico pela Cirurgia e Traumatologia Bucomaxilofaciais e Neurocirurgia sob anestesia geral e intubação orotraqueal. (Figura 3) Após antissepsia extraoral e aposição dos campos cirúrgicos estéreis, foi realizado infiltração anestésica com lidocaína 2\% e epinefrina 1:100.000 em região do canto medial da órbita. Foi realizado uma incisão vertical circundando o canto medial da órbita esquerda e o contorno nasal, nomeada como acesso de Lynch, estendendo-se desde a glabela a asa do nariz ipsilateral. Em seguida, foi realizado o descolamento dos tecidos por planos para exposição do projétil, preservando o ducto lacrimal ipsilateral. A bala foi removida com o auxílio de pinças hemostáticas. Após a remoção do corpo estranho, foi realizado irrigação copiosa do local com soro fisiológico $0,9 \%$ e inspeção visual da região, a qual não foi detectado hemorragia ou extravasamento do líquido cefalorraquidiano (LCR). Em seguida, foram colhidos 08 tubos de 4ml de sangue autógeno e condicionados em centrífuga. O conteúdo I-PRF foi introduzido em sítio cirúrgico acessado e realizado sutura dos tecidos por 
planos, sendo os pontos internos com fio vicryl 4-0 e a pele com fio nylon 4-0. O paciente evolui com 03 meses de pósoperatório com amaurose ocasionada pelo trauma e oftalmoplegia do olho esquerdo, sem queixas respiratórias ou neurológicas. (Figura 1)

Figura 2. Figura A:Reconstrução coronal da TC pré-operatória, evidenciando corpo estranho hipodenso dentro do seio esfenoidal. Figura B e C:Corte sagital e axial da TC pré-operatória, respectivamente, evidenciando fratura da região nasal, orbital, etmoidal e esfenoidal. Figura D: Reconstrução 3D evidenciando projétil alojado no canto medial do olho. Figura E, F e G: Reconstrução coronal, corte sagital e axial da TC pós-operatória, respectivamente, evidenciando área radiolúcida em região dos ossos esfenoidal, etmoidais e orbitária do OE, globos oculares íntegros. Figura F: Reconstrução em 3D da TC pósoperatória evidenciando ausência de projétil.
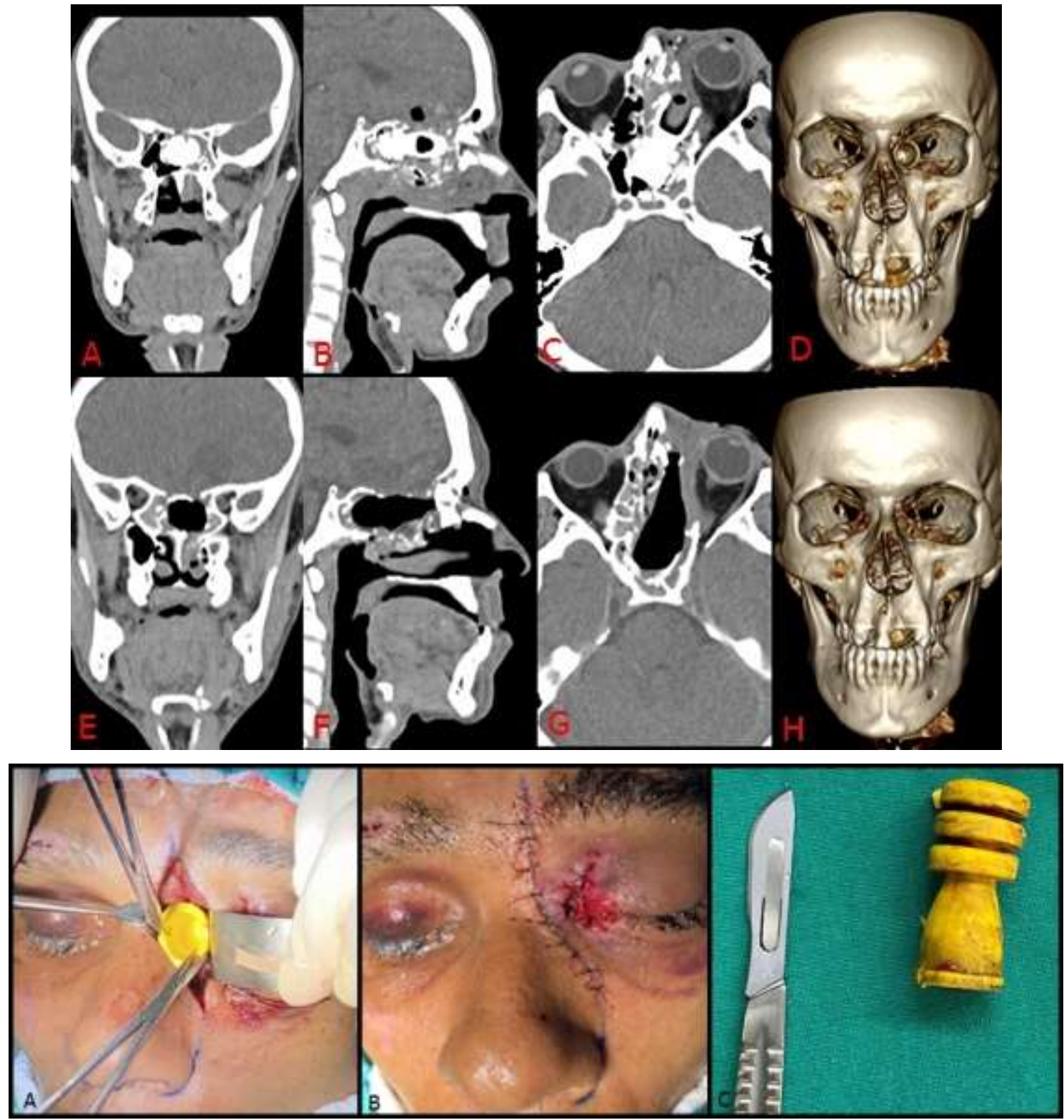

Fonte: Autores.

Figura 3: Figura A: Aspecto clínico extraoral, evidenciando acesso para-nasal (região do canto medial do OE), após divulsão dos tecidos e acesso ao projétil, aprisionado com pinças hemostáticas. Figura B: Aspecto final após a remoção da bala de 
borracha, evidenciando suturas extraoral em posição e ferida em cicatrização por segunda intenção (compatível com entrada do projétil). Figura C: Corpo estranho compatível com bala de borracha após a remoção.

\section{Discussão}

Os projéteis confeccionados com material borrachóide são classificados como "arma menos letal" ou "não letal" e são comumente utilizados para incapacitar ou limitar indivíduos perigosos e controlar multidões, a fim de evitar o uso de arma de fogo convencional ou "arma letal". Apesar de não ser caracterizada como uma arma letal, a bala de borracha pode ser fatal ou gerar sequelas importantes no indivíduo acometido. Essas complicações dependem da energia cinética, do agente biológico e da energia eletromagnética da bala de borracha utilizada. Outros fatores influentes nas lesões faciais são: a massa, o tamanho, a forma e a velocidade do impacto, bem como da natureza dos tecidos que a bala de borracha atinge. As balas de ponta redonda em alta velocidade podem causar ferimentos penetrantes, diferente das balas de ponta chata que tem maior probabilidade de causarem ferimentos contundente. ${ }^{56}$ (Lavy; Abu Asleh, 2003)(Kobayashi; Mellen, 2009) Dentre os fatores que afetam a penetração e a gravidade da lesão por bala de borracha estão: o limite elástico e a viscosidade da área do corpo lesada. Em uma área com baixo limite elástico, como os ossos finos da face, lesões penetrantes graves são frequentemente detectadas. O dano aos tecidos moles provocado pela bala de borracha é causado por uma compressão direta dos tecidos moles pela ponta contusa da bala e pelas ondas de choque que são geradas no momento do impacto, podendo criar uma laceração e ocasionar fraturas locais. (Ueeck et al., 2007)( Kalantar Motamedi et al, 2013) Os traumas localizados no terço médio da face exigem uma avaliação da neurocirurgia, oftalmologia, cirurgia e traumatologia bucomaxilofaciais, cirurgião vascular e otorrinolaringologista. A solicitação de exames complementares são fundamentais no diagnóstico. A tomografia computadorizada é essencial no diagnóstico, pois auxilia na identificação, bem como no curso e localização da bala de borracha e dos ferimentos internos acometidos. O uso do contraste torna-se relevante visto que a face possui estruturas nobres como vasos sanguíneos que podem ter sido lesados no trajeto do corpo estranho. As balas alojadas na zona III de Manson (delimitada entre o ângulo mandibular e a base do crânio), mesmo em paciente estáveis, porém, sintomáticos, a angiografia pode ser indicada para o diagnóstico preciso de lesões vasculares e se necessário embolização de vasos. (Glana et al., 2007) (Melo et al, 2017) A órbita foi o local mais acometido por lesões com bala de borracha, causando fratura orbitária e a principal sequela relatada na literatura foi dano oftalmológico como pode ser observado na Tabela I. O presente relato corrobora com os estudos, visto que a lesões por bala de borracha, apesar de parecerem inofensivas e serem classificadas como armas não letais, quando atingem a região facial podem causar complicações tardias (amaurose, paralisia faciais, cicatrizes) ou óbito. No caso relatado, o paciente evolui com amaurose como sequela. (Amaral et al., 2017) 
Tabela 1. Tabela evidenciando gênero, idade, fratura facial e seu respectivo tratamento, sequela e morte relatada na literatura entre os anos de 1975 e 2017. *Uma morte foi relatada, mas a causa não foi estabelecida, suspeita-se de obstrução das vias aéreas ou lesão torácica. (Millar et al., 1975)

\begin{tabular}{|c|c|c|c|c|c|}
\hline \multicolumn{6}{|c|}{ LESÕES FACIAIS POR BALA DE BORRACHA (1975-2017) } \\
\hline ARTIGO & SEXO/IDADE & IDADE & $\begin{array}{l}\text { FRATURA } \\
\text { FACIAL }\end{array}$ & $\begin{array}{l}\text { TRATAMENTO DE } \\
\text { FRATURA FACIAL }\end{array}$ & SEQUELA/MORTE \\
\hline \multirow[t]{3}{*}{$\begin{array}{c}\text { AMARAL et } \\
\text { al,; } 2017\end{array}$} & Masculino & 13 & $\begin{array}{c}\text { Mandíbula (corpo, } \\
\text { ângulo e ramo) }\end{array}$ & Osteossíntese & $\begin{array}{c}\text { Sem sinais de infecção } \\
\text { ou paralisia facial }\end{array}$ \\
\hline & Masculino & 17 & $\begin{array}{l}\text { Órbita, maxila e } \\
\text { ossos próprios do } \\
\text { nariz }\end{array}$ & Osteossíntese & Evisceração do olho \\
\hline & Feminino & 29 & $\begin{array}{c}\text { Mandíbula (corpo, } \\
\text { ângulo e ramo) }\end{array}$ & Osteossíntese & $\begin{array}{l}\text { Osteomielite do osso } \\
\text { mandibular }\end{array}$ \\
\hline $\begin{array}{l}\text { LAVY; ABU } \\
\text { ASLEH, } 2003\end{array}$ & 14 pacientes & $\begin{array}{l}\text { Média } 25 \\
\text { anos } \\
\text { (4-60anos) }\end{array}$ & 14 Fratura órbita & $\begin{array}{c}4 \text { reconstruções da } \\
\text { órbita }\end{array}$ & Alterações oculares \\
\hline $\begin{array}{c}\text { MILLAR et } \\
\text { al,; } 1975\end{array}$ & 32 pacientes & $7-67$ anos & $\begin{array}{c}12 \text { zigoma } \\
2 \text { maxilas } \\
5 \text { órbitas } \\
11 \text { nasais } \\
2 \text { mandíbulas }\end{array}$ & Não relata & $\begin{array}{l}\text { Deformidade facial } \\
\text { Anosmia } \\
\text { Alteração ocular } \\
1 \text { morte* }\end{array}$ \\
\hline \multirow{5}{*}{$\begin{array}{l}\text { KHONSARI } \\
\text { et al,; } 2010\end{array}$} & Feminino & 48 & $\begin{array}{l}\text { Fratura do osso } \\
\text { zigomático e } \\
\text { assoalho orbitário }\end{array}$ & $\begin{array}{c}\text { Prótese ocular e } \\
\text { reconstrução de tecidos } \\
\text { moles }\end{array}$ & Perda ocular \\
\hline & Masculino & 20 & $\begin{array}{c}\text { Fratura do assoalho } \\
\text { orbitário }\end{array}$ & $\begin{array}{c}\text { Reconstrução orbitária } \\
\text { e prótese ocular }\end{array}$ & $\begin{array}{c}\text { Infecção, perda ocular, } \\
\text { rinorréia purulenta e } \\
\text { epífora }\end{array}$ \\
\hline & Feminino & 45 & Não relata & - & - \\
\hline & Masculino & 43 & Fratura orbitária & Reconstrução orbitária & Infecção \\
\hline & Masculino & 44 & $\begin{array}{l}\text { Fratura de } \\
\text { mandíbula }\end{array}$ & $\begin{array}{l}\text { Osteossíntese de } \\
\text { fratura mandibular }\end{array}$ & $\begin{array}{l}\text { Paralisia facial e } \\
\text { infecção }\end{array}$ \\
\hline $\begin{array}{l}\text { BALOURIS } \\
\text { et al,; } 1990\end{array}$ & Não relata & $\begin{array}{l}\text { Idade média: } \\
18 \text { anos }\end{array}$ & $\begin{array}{l}10 \text { fraturas } \\
\text { orbitárias }\end{array}$ & Não relata & $\begin{array}{l}\text { Enucleação ocular, } \\
\text { amaurose, déficit de } \\
\text { visão }\end{array}$ \\
\hline $\begin{array}{l}\text { GROSS et al,; } \\
2005\end{array}$ & Masculino & 32 & $\begin{array}{l}\text { Fratura da parede } \\
\text { anterior do seio } \\
\text { maxilar }\end{array}$ & $\begin{array}{c}\text { Remoção por via } \\
\text { endoscópica transnasal }\end{array}$ & Não relata. \\
\hline
\end{tabular}

Fonte: Autores. 
Nos casos de acometimento direito ou indireto da cavidade orbitária, podem ser necessárias condutas de emergência. A cirurgia oftalmológica imediata está indicada para os casos de lesões de globo aberto, lesões do sistema nasolacrimal ou fratura da parede orbital com compressão muscular que podem ser facilmente identificadasnos serviços de emergência de atendimento pré-hospitalar (que podem investigar tônus ocular, lesão de globo, percepção de luz, reatividade pupilar ou oculomotricidade). O tratamento cirúrgico precoce dessas lesões é necessário para melhorar o prognóstico visual, os resultados estéticos e diminuir o risco de endoftalmite, diplopia binocular e epífora crônica. Um acompanhamento a longo prazo é necessário para corrigir possíveis sequelas. (Lartizien, et al., 2019) As infecções após a remoção ou não do projétil são relatadas, principalmente por não ser estéril. Sua cápsula de borracha pode se desintegrar do restante do corpo estranho gradualmente e liberar um material tóxico local, podendo ser irritante aos tecidos ao redor, como ao nervo facial. (Gaborian et al., 1998) A presença de infecção após a abordagem inicial para remoção do corpo estranho pode estar associada devido a permanência de estilhaços no local. A reabordagem cirúrgica para remoção completa no material e antibioticoterapia se mostraram eficaz na literatura quando presente. (Khonsari et al., 2010) Nos seios paranasais, a presença da bala de borracha pode ocasionar várias complicações dentre elas: sinusite crônica, fistula cutânea, formação de rinólito, meningite e dor crônica. Nesses casos, recomenda-se que a bala de borracha seja removida com segurança sem danos excessivos as estruturas vitais ao redor. (Bloom et al., 2020) No presente caso, a bala de borracha foi removida, a qual durante seu percurso fraturou os seios etmoidais e esfenoidal, mantendo-se alojada no seio esfenoidal, abaixo da sela turca. A decisão da remoção foi realizada com o objetivo de prevenir complicações já supracitadas. Em todos os casos apresentados na Tabela I a bala de borracha foi removida. A endoscopia nasossinusal pode ser utilizada para remoção da bala de borracha dos seios da face, fornecendo um acesso menos invasivo e excelente visualização.O acesso de Caldwell-Luc e etmoidectomia externa são descritas para remoção de corpos estranhos alojados no seio maxilar e etmoidal, respectivamente. (Ballantyne, 2006) Deve-se ter cautela na remoção da bala de borracha alojada no seio esfenoidal. Estruturas nobres como a artéria carótida interna e nervo óptico estão próximos ao local e podem ser lesionados, bem como, o possível extravasamento de líquido cefalorraquidiano. (Mclean et al., 2005) A sistematização do tratamento de feridas envolve uma abordagem com protocolos adequados para avaliação clínica e manejo do tratamento pelas barreiras que interferem na cicatrização, incluindo a presença de tecido necrótico, células senescentes, matriz extracelular alterada, hipóxia, excesso de bactérias, biofilme, e enzimas inflamatórias(Al-ahmady et al, 2018). A fibrina rica em plaquetas consiste em uma matriz onde citocinas, fatores de crescimento e plaquetas que são retidas através de um processo de centrifugação controlada pelo próprio sangue venoso do paciente, o qual, não necessita de aditivos bioquímicos. Constitui em um processo simples e econômico, oferecendo cicatrização para a ferida, atuando como uma estrutura biodegradável para a entrega de fatores de crescimento, síntese de colágeno e angiogênese. (Karla et al., 2020) O plasma rico em fibrina (PRF) consiste em uma matriz na qual citocinas, fatores de crescimento e células plaquetárias são retidas e podem ser liberados lentamente, oferecendo os elementos necessários para a cicatrização de feridas, atuando como uma estrutura biodegradável. Além disso, é considerado um procedimento seguro, de curto tempo de fabricação e implementação. Pode ser utilizada para promover a cicatrização de feridas, estabilizações de enxertos, regeneração óssea, selamento de feridas e hemostasia, através da capacidade da fibrina rica em plaquetas induzir a proliferação de todos os tipos de células, especialmente osteoblastos, sugerindo que os fatores de crescimento promovem angiogênese, proliferação e diferenciação celular. Após o processo de centrifugação, a matriz de fibrina é capaz de direcionar com eficiência a migração de células-tronco. (Karla et al., 2020)

\section{Conclusão}

As lesões penetrantes ocasionadas por bala de borracha na face devem ser tratadas por uma equipe multidisciplinar para diagnosticar e definir o tratamento com melhor prognóstico. Tais feridas devem ser tratadas como feridas ocasionadas por arma de fogo, pois podem ocasionar complicações tais qual, bem como, deixar sequelas importantes. A tomografia 
computadorizada é essencial no diagnóstico e decisão de tratamento, em conjunto com o exame clínico multidisciplinar. O método cirúrgico mais invasivo ou conservador irá depender da localização da bala de borracha, bem como sua proximidade com estruturas nobres.

\section{Referências}

Al-Ahmady, H. H., El Sayed, M., Fereir, A., Ekram, A., \& Mousa, K. (2018). Removal of large impacted foreign body from the base of the skull through submandibular access: A multidisciplinary approach. International journal of surgery case reports, 50, 21-24. https://doi.org/10.1016/j.ijscr.2018.07.012

Amaral, M. B. F. et al (2017). Facial fractures caused by less-lethal rubber bullet weapons: case series report and literature review. Oral and Maxillofacial Surgery, 21(3), 357-361.

Ballantyne B. (2006). Medical management of the traumatic consequences of civil unrest incidents: causation, clinical approaches, needs and advanced planning criteria. Toxicological reviews, 25(3), 155-197. https://doi.org/10.2165/00139709-200625030-00003

Bloom, W. R., \& Bloom, T. D. (2020). Epidemiology of Eye Injuries Caused by Personal Protection Devices and Kinetic Impact Projectile Weapons. JAMA ophthalmology, 138(12), 1318-1319. https://doi.org/10.1001/jamaophthalmol.2020.4434

Gaboriau, H. P., \& Kreutziger, K. L. (1998). Penetrating injuries of the face. The Journal of the Louisiana State Medical Society: official organ of the Louisiana State Medical Society, 150(1), 6-9.

Glapa, M., Kourie, J. F., Doll, D., \& Degiannis, E. (2007). Early management of gunshot injuries to the face in civilian practice. World journal of surgery, 31(11), 2104-2110. https://doi.org/10.1007/s00268-007-9220-2

Gross, M. et al. (2005). Penetrating rubber bullet into the ethmoid sinus: Should the bullet be removed? Otolaryngology - Head and Neck Surgery. 133(5), 814-6.

Kalantar Motamedi MH. Comprehensive Management of Maxillofacial Projectile Injuries at the First Operation; "Picking up the Pieces". Trauma Mon. 2013 Winter; 17(4):365-610.5812/traumamon.9279.

Karla, C. et al. (2020) Matriz autóloga de fibrina rica em plaquetas em ambientes de tratamento de feridas : uma revisão sistemática de ensaios clínicos randomizados. 11(2),

Khonsari, R. H. et al (2010). Severe facial rubber bullet injuries: Less lethal but extremely harmful weapons. Injury, 41(1), 73-76

Kobayashi, M. \& Mellen, P. F. (2009). Rubber bullet injury: Case report with autopsy observation and literature review. American Journal of Forensic Medicine and Pathology, 30(3), 262-7.

Lartizien, R. et al. (2019) Yellow vests protests: facial injuries from rubber bullets. The Lancet, 394(10197), 469-470.

Lavy, T.; Abu Asleh, S. (2003). Ocular rubber bullet injuries. Eye, 17(7), 821-824,.

McLean, J. N., Moore, C. E., \& Yellin, S. A. (2005). Gunshot wounds to the face--acute management. Facial plastic surgery: FPS, 21 (3), 191-198. https://doi.org/10.1055/s-2005-922859

Melo, M. N., Pantoja, L. N., de Vasconcellos, S. J., Sarmento, V. A., \& Queiroz, C. S. (2017). Traumatic Foreign Body into the Face: Case Report and Literature Review. Case reports in dentistry, 2017, 3487386. https://doi.org/10.1155/2017/3487386

Millar, R. et al. (1975). Injuries caused by rubber bullets: A report on 90 patients. British Journal of Surgery, 62(6), 480-486.

Motamedi M. H. (2007). Primary treatment of penetrating injuries to the face. Journal of oral and maxillofacial surgery: official journal of the American Association of Oral and Maxillofacial Surgeons, 65(6), 1215-1218. https://doi.org/10.1016/j.joms.2007.03.001

Pereira A. S. et al. (2018). Metodologia da pesquisa científica. UFSM. 5.3) Koche, J. C. (2011). Fundamentos de metodologia científica. Petrópolis: Vozes. Disponível em: http://www.brunovivas.com/wp content/uploads/sites/10/2018/07/K\%C3\%B6che-Jos\%C3\%A9-Carlos0D0AFundamentos-de-metodologiacient\%C3\%ADfica-_teoria-da0D0Aci\%C3\%AAncia-e-inicia\%C3\%A7\%C3\%A3o-\%C3\%A0pesquisa.pdf https://repositorio.ufsm.br /bitstream/handle/1 /15824/Lic_Computacao_Metodologia-Pesquisa-Cientifica.pdf?sequence=1

Rodríguez, Á., Peña, S., Cavieres, I., Vergara, M. J., Pérez, M., Campos, M., Peredo, D., Jorquera, P., Palma, R., Cortés, D., López, M., \& Morales, S. (2020). Ocular trauma by kinetic impact projectiles during civil unrest in Chile. Eye (London, England), 1-7. Advance online publication. https://doi.org/10.1038/s41433-020-01146-w

Ueeck B. A. (2007). Penetrating injuries to the face: delayed versus primary treatment--considerations for delayed treatment. Journal of oral and maxillofacial surgery : official journal of the American Association of Oral and Maxillofacial Surgeons, 65(6), 1209-1214. https://doi.org/10.1016/j.joms.2006.10.078 